\title{
Incidencia y factores asociados para infarto agudo de miocardio en pacientes con dolor torácico
}

\author{
Incidence and associated factors for acute myocardial infarction in patients \\ with chest pain
}

Leidy Henao P.? , Laura Bustos', Sebastián Salazar', Luis Felipe Cárdenas L. ${ }^{2}$, Giovanni Caviedes P. ${ }^{3}$

\section{Resumen}

La enfermedad coronaria es una de las principales patologías generadoras de morbilidad y mortalidad a nivel mundial; a nivel nacional hay pocos estudios publicados que sugieran cuáles son las características semiológicas que se asocian a Infarto Agudo de Miocardio (IAM) en un paciente que consulta por dolor torácico.

Pacientes y Métodos: Estudio observacional, prospectivo tipo de casos y controles anidado en una cohorte. Definición de caso: Pacientes que consultaron por dolor torácico y se les diagnóstico Infarto Agudo de Miocardio (IAM). Definición de control: Pacientes con cuadro de dolor torácico causado por otras patologías diferentes al IAM. Se tomaron dos controles por cada caso. La recolección de los datos se realizó mediante un instrumento, aplicado a través de la entrevista y la revisión de historias clínicas. El análisis se realizó a través del paquete estadístico Epi Info7.0.

Resultados: Total de 141 pacientes, 47 casos y 94 controles. El 93.6\% ingresó por dolor torácico. Se muestra una asociación entre la presencia de ciertas variables clínicas y factores de riesgo cardiovascular y la probabilidad de IAM. Dentro de los Factores significativos estadísticamente para riesgo cardiovascular se encontró la Edad y tabaquismo. Conclusión: Con base en los hallazgos de este trabajo se concluye que existen algunas características clínicas y factores de riesgo que al estar presentes en pacientes que consultan por dolor torácico y/o equivalente anginoso, pueden orientar al clínico hacia un diagnóstico de IAM.

Palabras clave: Dolor torácico, equivalente anginoso, infarto agudo de miocardio, factores de riesgo.

\begin{abstract}
Coronary heart disease is one of the main pathologies causing morbidity and mortality worldwide. In Colombia there are few published studies showing the semiological characteristics associated with acute myocardial infarction (AMI) in a patient with chest pain.

Patients and Methods: A case prospective observational study and controls nested in a cohort. Case definition: Patients with chestpain and diagnosed with acute myocardial infarction (AMI). Definition of control: Patients with chest pain caused by pathologies other than AMI. Two controls were taken for each case. Data collection was performed usingan instrumenta pplied by interview andreview of medical histories. The analysis was performe dusing the statistical package Epi Info 7.0.

Results: Total of 141 patients, 47 cases and 94 controls. $93.6 \%$ was admitted for chest pain. There is an association between the presence of certain clinical variables and cardiovascular risk factors and the likelihoodof AMI. Age and smoking are among thestatistically significant factorsfor cardiovascular risk.

Conclusion: Based on the findings it is concluded that there are some clinical features and risk factors that, when presentin patients with chest pain and /or angina equivalent, can lead the clinician to a diagnosis of AMI.
\end{abstract}

Key words: Chest pain, anginal equivalent, acute myocardial infarction, risk factors.

\section{Introducción}

El dolor torácico agudo es responsable del 5-20\% de las urgencias médicas hospitalarias. De los pacientes que acuden a Urgencias por dolor torácico, solo una pequeña minoría tiene una condición que

1 Médico, Universidad Surcolombiana

2 Médico internista. Abogado. Docente Universidad Surcolombiana

3 Médico internista. Farmacólogo Clínico. Epidemiólogo. Docente Universidad Surcolombiana. Grupo de investigación Desarrollo Social, Salud Pública y Derechos Humanos. Correspondencia: Correo electrónico: giocape@hotmail.com

Recibido: 08/10/2013 - Revisado: 20/12/2013 - Aceptado: 07/09/2014 
Incidencia y factores asociados para infarto agudo de miocardio en pacientes con dolor torácico/Leidy Henao P., et al.
R.F.S Revista Facultad de Salud

Julio - Diciembre de 2014;6(2): 32-38 ponga en peligro la vida. El $60 \%$ de los dolores torácicos no son de origen cardiaco, ya que aproximadamente el 36\% son de origen musculo esquelético, el $11 \%$ corresponde a angina estable y solo un $1.5 \%$ corresponde a angina inestable o Infarto agudo de miocardio ${ }^{[1]}$ El infarto agudo de miocardio hace referencia a la necrosis de las células del músculo cardíaco producida por la isquemia de una zona del corazón secundaria a la obstrucción de las arterias coronarias ${ }^{[2]}$.

Es fundamental reconocer estas situaciones que necesitan una actuación inmediata y diferenciarlas de las entidades benignas que pueden darse de alta directamente de los Servicios de Urgencias, evitando dos problemas frecuentes, el subdiagnóstico y la sobreinternación. El primero con el riesgo de dar de alta un síndrome coronario agudo y el segundo con la realización de pruebas complementarias innecesarias y ocupación de camas de hospitalización con pacientes que no lo necesitan. Las estrategias de manejo del dolor torácico deben basarse en el diagnóstico rápido y en una estratificación del riesgo adecuada, basada fundamentalmente en la presencia de enfermedad aterosclerótica previa, factores de riesgo cardiovascular y las características del dolor torácico, con el apoyo de la exploración física y de las pruebas complementarias, fundamentalmente el electrocardiograma y la determinación analítica de marcadores de daño miocárdico (troponina I). Los pacientes con antecedentes de patología aterosclerótica previa, como enfermedad coronaria, cerebrovascular y/o vascular periférica tienen, de inicio, una mayor probabilidad de que su dolor torácico se deba a un síndrome coronario agudo.

La aterosclerosis y sus complicaciones son la principal causa de morbimortalidad en los países desarrollados. En Estados Unidos 60 millones de adultos padecen enfermedad cardiovascular, la cual es responsable de $42 \%$ de todas las muertes cada año ${ }^{[3]}$. Los factores de riesgo más importantes para la presentación de síndrome coronario agudo son fácilmente medibles y potencialmente modificables. Responden por una gran proporción del riesgo en (más de $90 \%$ ) para un infarto agudo de miocardio. Los efectos de estos factores de riesgo son más fuertes en hombres jóvenes (riesgo atribuible [RA] cercano a 93\%) y mujeres (RA aproximado de $96 \%$ ) indicando que, incluso pueden prevenirse los IAM más prematuros. En todo el mundo, los dos más importantes factores de riesgo son el atribuible de un IAM. Factores psicosociales, obesidad abdominal, diabetes e hipertensión arterial fueron los siguientes factores de riesgo más importantes en hombres y mujeres, pero su efecto relativo varia en diferentes regiones del mundo ${ }^{[4]}$.

Este estudio fue realizado con el objetivo de identificar los factores de riesgo cardiovascular y las características clínicas que se asocian a IAM en los pacientes que consultaron al servicio de urgencias del Hospital Universitario de Neiva por dolor torácico y/o equivalente anginoso en un período de 7 meses. De igual forma se busca caracterizar el manejo médico instaurado a los pacientes.

Debido a la alta prevalencia de factores de riesgo cardiovascular en la población general, es indispensable realizar estudios que aclaren cuáles son los factores de riesgo asociados a infarto agudo de miocardio en la población de pacientes que consultan al servicio de urgencias por dolor torácico y/o equivalente anginoso.

\section{Materiales y métodos}

\section{Definiciones}

Definición de equivalente anginoso ${ }^{[4]}$ : Son los síntomas diferentes a los convencionales del dolor torácico por síndrome coronario agudo tales como: sincope, disnea súbita, opresión precordial postprandial, dolor en maxilar inferior, sensación de atadura en codos y muñecas.

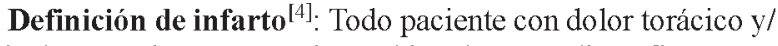
o equivalente anginoso, con o sin cambios electrocardiográficos compatibles con isquémia y con troponina I positiva.

Definición de caso: Todo paciente que consultó al servicio de urgencias por dolor torácico y/o equivalente anginoso, cuya causa demostrada fue un Infarto Agudo de Miocardio (IAM).

Definición de control: Aquel paciente que consultó al servicio de urgencias por dolor torácico y/o equivalente anginoso, al cual se le descartó infarto con troponina I de alta sensibilidad negativa. De cada uno de los casos encontrados en el periodo del estudio se eligió de manera aleatoria 2 controles.

Tipo de estudio: Se realizó un estudio observacional prospectivo, del tipo de casos y controles anidado en una cohorte en un periodo de 7 meses en el servicio de Urgencias del Hospital Universitario Hernando Moncaleano Perdomo de Neiva.

Muestra: Se tomaron la totalidad de pacientes que consultaron por dolor torácico y/o equivalente anginoso al servicio de urgencias en el periodo de estudio y que cumplieron con los criterios de inclusión y los de exclusión; posteriormente esta muestra fue dividida en dos grupos: aquellos pacientes a los cuales se les documentó un IAM (casos) y aquellos pacientes que presentaron dolor torácico y/o equivalente anginoso por alguna causa diferente de IAM (controles). Se tomaron todos los casos y de manera aleatoria se tomaron 2 controles por cada caso.

\section{Criterios de inclusión:}

1. Ser mayor de 14 años.

2. Pacientes con diagnóstico de ingreso de dolor torácico y/o equivalente anginoso.

3. Que se le haya tomado troponina I dentro de su estudio diagnóstico.

\section{Criterios de exclusión:}

1. Dolor torácico secundario a trauma.

2. Dolor torácico iatrogénico.

3. Pacientes con antecedentes de enfermedad psiquiátrica

4. Pacientes con antecedentes de enfermedades genéticas y/o retraso mental

5. Pacientes que ingresaron por intoxicación.

6. Pacientes con dificultad para la expresión verbal

La recolección de los datos fue llevada a cabo por los investigadores mediante la consignación de la información proveniente de una entrevista con el paciente y de la historia clínica en un instrumento previamente diseñado, cuyo diligenciamiento no tomó más de 15 minutos. Los pacientes se siguieron diariamente desde el ingreso al servicio de urgencias hasta su egreso de la institución.

La recolección de los datos se realizó de la siguiente manera: A todo paciente que consultó al servicio de Urgencias y cumplía con los criterios de inclusión y exclusión se le diligenció un formulario el cual contenía datos demográficos, valor de Troponina I, características del segmento ST en el EKG, características semiológicas del cuadro clínico, presencia de síntomas acompañantes, presencia de factores de riesgo cardiovascular entre otros. Se tomaron los resultados de la curva enzimática (Troponina I de ingreso, de las 6 horas y las 12 horas). Con el seguimiento hasta el alta hospitalaria se recolectaron otros datos como: resultados del cateterismo cardíaco, del ecocardiograma, estancia hospitalaria en 
R.F.S Revista Facultad de Salud

Julio - Diciembre de 2014;6(2): 32-38
Incidencia y factores asociados para infarto agudo de miocardio en pacientes con dolor torácico / Leidy Henao P., et al. días, mortalidad al egreso, etiología del cuadro clínico (diagnóstico) y tratamiento al egreso.

Los datos registrados en el instrumento se pasaron a una hoja de cálculo de Excel 2007 y se procedió a cargarla en el paquete estadístico Epi Info 7.0, realizando el siguiente análisis:

- Un análisis univariado para realizar la descripción de la población, a las variables cualitativas se les calculó la distribución de frecuencias y porcentajes. Posteriormente se utilizaron estos datos para hacer una descripción estadística comparativa entre las características de la población de los casos y la de los controles.

- Un análisis bivariado para establecer la asociación entre las características clínicas y la presencia de cada uno de los factores de riesgo en estos pacientes con la probabilidad de IAM como causante del cuadro inicial que llevó al paciente a consultar. Este se realizó a través de la construcción de tablas de $2 \times 2$ calculando el OR (odds ratio) a cada una de las variables y analizando los resultados con la prueba chi cuadrado de Pearson, la cual se consideró estadísticamente significativa cuando $p<0,05$. En los casos en que las frecuencias de algunas de las variables fueran menor de cinco, los resultados se analizaron bajo la prueba exacta de Fisher.

\section{Consideraciones éticas}

Según lo descrito en la resolución 8430 de 1993, este estudio es clasificable como una investigación sin riesgo, por su naturaleza y características metodológicas. Sin embargo en todos los casos se obtuvo el consentimiento verbal de cada uno de los participantes explicando previamente de manera clara los objetivos del estudio. El paciente se encontraba en la libertad de retirar su consentimiento y abandonar el estudio en cualquier momento.

Para la realización de este estudio fue necesaria la aprobación del mismo por el centro de investigación y extensión del Hospital Universitario de Neiva, quienes decidieron que al ser este un estudio con riesgo nulo para el paciente puesto que no se realizaría ninguna intervención directa sobre él que pudiera afectar su integridad física o emocional. Los investigadores se comprometen a mantener los resultados y las bases de datos bajo estricta confidencialidad, la custodia de la información de los pacientes estará conforme a lo dispuesto por la resolución 1715 de 2005, Ley 23 de 1981 y resolución 1995 de 1999.

\section{Resultados}

Durante el período de estudio, 250 pacientes cumplieron con los criterios de inclusión y de exclusión, por lo tanto fueron ingresados dentro del estudio. De los pacientes entrevistados, 47 correspondían a casos de IAM y los otros 203 correspondían a potenciales controles; de manera aleatoria se escogieron 2 controles por cada caso de IAM como se tenía planeado.

De los 141 pacientes que entraron en el estudio, 72 eran mujeres $(51,1 \%)$. La mediana de edad de esta población fue de 62 años con un rango intercuartílico de edad entre 54 y 71 años. La mayoría de pacientes $(n=123)$ eran procedentes del área urbana $(87,2 \%)$. La mediana de tiempo en horas desde el inicio de los síntomas hasta la consulta fue de 5,2 (RI: 2-24) para los casos y de 11 (2,5-60) para los controles. La predominancia de género de los pacientes en cada grupo fue diferente, mientras en el grupo de los controles la mujeres fueron el $54,3 \%$ en el grupo de los casos la mayoría de los pacientes eran hombres $(61,7 \%$ ) (Tabla 1 ).

Tabla 1. Características sociodemográficas de los pacientes que consultaron al servicio de urgencias del Hospital Universitario de Neiva por dolor torácico y/o equivalente anginoso.

\begin{tabular}{|c|c|c|}
\hline Variable & Casos & Controles \\
\hline Masculino, n (\%) & $29(61,7)$ & $43(45,7)$ \\
\hline Femenino, n (\%) & $18(38,3)$ & $51(54,3)$ \\
\hline Edad (años), Mediana y RI & $64(56,5-71,5)$ & $60(52,5-71)$ \\
\hline Estancia (días), Mediana y RI & $6,5(3-15)$ & $6(3-11,5)$ \\
\hline Tiempo desde inicio de síntomas a la consulta(hrs), Mediana y RI & $5,2(2-24)$ & $11(2,5-60)$ \\
\hline \multicolumn{3}{|l|}{ Factores de riesgo cardiovascular, n (\%) } \\
\hline Edad (H ${ }^{3} 45$ años; $M{ }^{3} 55$ años) & $45(95,7)$ & $70(74,5)$ \\
\hline Hipertensión arterial & $27(57,4)$ & $56(59,6)$ \\
\hline Diabetes Mellitus & $8(17)$ & $17(18,1)$ \\
\hline Dislipidemia & $16(34)$ & $41(43,6)$ \\
\hline Sedentarismo & $19(40,4)$ & $40(42,6)$ \\
\hline Tabaquismo & $21(44,7)$ & $22(23,4)$ \\
\hline Obesidad abdominal & $29(61,7)$ & $59(62,8)$ \\
\hline Antecedentes familiares de enfermedad coronaria prematura & $4(8,5)$ & $9(9,6)$ \\
\hline Antecedentes personales de enfermedad coronaria & $6(12,8)$ & $24(25,5)$ \\
\hline No se identifico factor de riesgo & 0 & $5(5,3)$ \\
\hline \multicolumn{3}{|l|}{ Mortalidad al egreso } \\
\hline $\mathrm{Si}$ & $5(10,6)$ & $1(1,1)$ \\
\hline No & $42(89,4)$ & $93(98,9)$ \\
\hline
\end{tabular}


Incidencia y factores asociados para infarto agudo de miocardio en pacientes con dolor torácico/Leidy Henao P., et al.
R.F.S Revista Facultad de Salud

Julio - Diciembre de 2014;6(2): 32-38
En el grupo de casos se observó una mortalidad al egreso en solo 5 pacientes mientras que en los controles fue en 1. (Tabla 1). En ambos grupos se encontró de manera similar la presencia de algunos factores de riesgo para enfermedad cardiovascular como: Hipertensión arterial (HTA), diabetes mellitus (DM), Sedentarismo, Obesidad abdominal y Antecedentes familiares de enfermedad coronaria prematura, tabaquismo y antecedentes personales de enfermedad coronaria. No se encontraron pacientes en el grupo de los casos con ausencia de factores de riesgo cardiovascular, mientras en el grupo de controles esta situación se presentó en el $5,3 \%$ (Tabla 1 ).
El 93.6\% de los pacientes ingresó al servicio de urgencias por dolor torácico, siendo la ubicación precordial la más frecuente con un $54,5 \%$ para los casos y un $59,1 \%$ para los controles. El tipo de dolor más común fue el opresivo con una presentación del 70,4\% en los controles comparada con los casos en tan solo un $25 \%$. Se evidencio una amplia variabilidad en la intensidad del dolor dentro de la Escala de valoración subjetiva de 1 a 10 tanto en los casos como en los controles. E1 34,1\% de los casos y el 42,1\% de los controles manifestaban un dolor no irradiado. La irradiación más frecuente fue el brazo izquierdo con un $36,4 \%$ y $17,1 \%$ para los casos y controles respectivamente seguido del cuello con un $20,4 \%$ y $11,4 \%$ para los casos y los controles respectivamente. (Tabla 2 ).

Tabla 2. Características Semiológicas del dolor torácico de los pacientes que consultaron al servicio de urgencias del Hospital Universitario de Neiva.

\begin{tabular}{|c|c|c|}
\hline Variable & Casos n (\%) & Controles n (\%) \\
\hline \multicolumn{3}{|l|}{ Dolor toráccico } \\
\hline $\mathrm{Si}$ & $44(93,6)$ & $88(93,6)$ \\
\hline No & $3(6,4)$ & $6(6,4)$ \\
\hline \multicolumn{3}{|l|}{ Localización } \\
\hline Retroesternal & $10(22,7)$ & $14(15,9)$ \\
\hline Precordial & $24(54,5)$ & $52(59,1)$ \\
\hline Interescapular & $1(2,3)$ & $1(1,1)$ \\
\hline Epigástrico & $2(4,5)$ & $12(13,6)$ \\
\hline Torácico difuso & $5(11,4)$ & $7(7,9)$ \\
\hline Otros & $2(4,5)$ & $3(3,4)$ \\
\hline \multicolumn{3}{|l|}{ Tipo de dolor } \\
\hline Urente & $7(15,9)$ & $5(5,7)$ \\
\hline Opresivo & $11(25)$ & $62(70,4)$ \\
\hline Sordo & $2(4,5)$ & $1(1,1)$ \\
\hline Punzada & $3(6,8)$ & $16(18,2)$ \\
\hline \multicolumn{3}{|l|}{ Intensidad } \\
\hline$(3 / 10)$ & $2(4,5)$ & $3(3,4)$ \\
\hline$(5 / 10)$ & $4(9,1)$ & $9(10,2)$ \\
\hline$(6 / 10)$ & $1(2,3)$ & $11(12,5)$ \\
\hline$(7 / 10)$ & $5(11,4)$ & $18(20,4)$ \\
\hline$(8 / 10)$ & $11(25)$ & $17(19,3)$ \\
\hline$(9 / 10)$ & $4(9,1)$ & $5(5,7)$ \\
\hline$(10 / 10)$ & $17(38,6)$ & $17(19,3)$ \\
\hline \multicolumn{3}{|l|}{ Irradiación del dolor } \\
\hline Brazo Izquierdo & $16(36,4)$ & $15(17,1)$ \\
\hline No irradia & $15(34,1)$ & $37(42,1)$ \\
\hline Hombro Izquierdo & $9(20,4)$ & $6(6,8)$ \\
\hline Cuello & $9(20,4)$ & $10(11,4)$ \\
\hline Interescapular & $7(15,9)$ & $22(25)$ \\
\hline Epigástrico & $7(15,9)$ & $9(10,2)$ \\
\hline Otra irradiación & $4(9,1)$ & $12(13,6)$ \\
\hline Ambos Brazos & $2(4,1)$ & $1(1,1)$ \\
\hline Brazo Derecho & $1(2,3)$ & $2(2,3)$ \\
\hline Ambos Hombros & $1(2,3)$ & $1(1,1)$ \\
\hline Mandíbula & $1(2,3)$ & $5(5,7)$ \\
\hline
\end{tabular}


R.F.S Revista Facultad de Salud

Julio - Diciembre de 2014;6(2): 32-38
Incidencia y factores asociados para infarto agudo de miocardio en pacientes con dolor torácico / Leidy Henao P., et al.
De los pacientes con Infarto agudo de miocardio $(n=47)$, solo el $21,3 \%$ presentaban cambios electrocardiog ráficos compatibles con elevación del ST (Figura 1). Los pacientes con Infarto agudo de miocardio egresaron del hospital con medicamentos como IECAs en un $88,1 \%$, Betabloqueadores en un $92,9 \%$, Estatinas en un $97,6 \%$ y ASA en el $100 \%$. Solo al $66,7 \%$ de estos pacientes se les prescribió Clopidogrel dentro del tratamiento ambulatorio (Figura 2).

Las variables semiológicas y los factores de riesgo fueron sometidos a la aplicación de un modelo matemático de regresión logística con el fin de buscar asociación entre estas y la presencia de IAM. Se encontró que la irradiación del dolor a

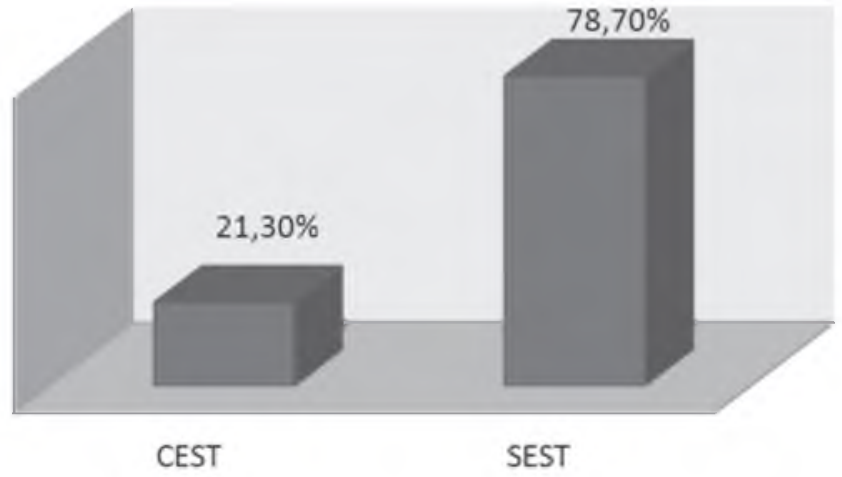

Figura 1. Segmento ST de los pacientes que consultaron al servicio de urgencias del Hospital Universitario de Neiva por dolor torácico y/o equivalente anginoso.

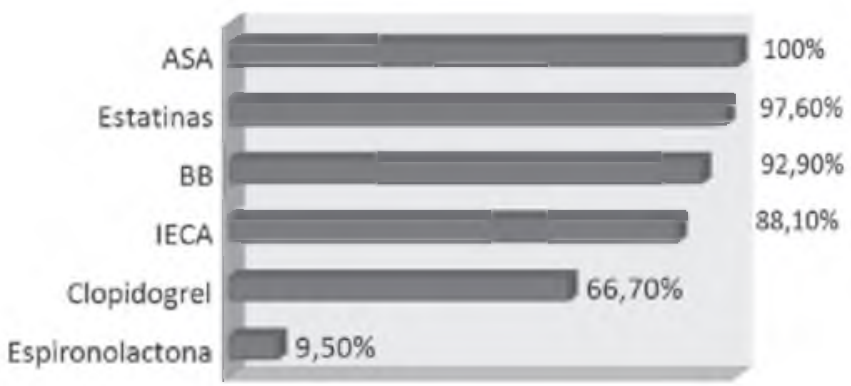

Figura 2. Tratamiento al egreso de los pacientes que consultaron al servicio de urgencias del Hospital Universitario de Neiva por dolor torácico y/o equivalente anginoso. brazo izquierdo, la irradiación del dolor a hombro izquierdo, la intensidad del dolor de 10/10, la duración del dolor entre 1 y 3 horas y la presencia de vómito; fueron variables estadísticamente significativas. También se encontró asociación entre IAM y factores de riesgo como: edad ( $\mathrm{H} \geq 45$ años; $\mathrm{M} \geq 55$ años) $y$ tabaquismo (Tabla 3 ).

\section{Discusión}

En este estudio se encontró que las características del cuadro clínico de los pacientes que cursan con IAM que se presentaron con mayor frecuencia son congruentes con las descritas en la literatura mundial, la cual hace referencia a un dolor opresivo de ubicación precordial, de intensidad máxima (10/10 en la escala subjetiva del dolor), irradiada principalmente a hombro y brazo izquierdo y acompañada de algunos síntomas autonómicos como: diaforesis, náuseas, palidez, palpitaciones, entre otros.

Dentro de las características clínicas que fueron significativas se identificó que la irradiación a brazo izquierdo aumentaba en 2.8 veces la probabilidad de que el paciente con dolor torácico estuviera cursando con IAM, al que lo hacía en 3,5 veces la irradiación a hombro izquierdo; estas dos características clínicas se encuentran reportadas en la literatura mundial cuando se describe la semiología clásica del IAM. Sin embargo Swap C. y colaboradores ${ }^{[5]}$ reportaron que la probabilidad de tener un infarto de miocardio cuando el dolor se irradia al hombro o brazo derecho es de 4,7 hallazgo que es muy superior al de nuestro estudio.

La presentación del dolor en su máxima intensidad (10/10 en la escala subjetiva del dolor) se asocia a un riesgo mayor a dos veces de que este sea causado por IAM. Si el paciente presenta un dolor que dura entre 1 a 3 horas la probabilidad de IAM es de 3,8 veces. La presencia de síntomas gastrointestinales asociados a IAM ha sido reportada en la literatura mundial por Autores como James L Meise ${ }^{[6]}$, el cual demuestra que los síntomas gastrointestinales asociados a IAM pueden estar presentes en un $35 \%$ de las personas Infartadas. Encontramos en nuestro estudio, que si el cuadro clínico inicial se encuentra acompañado de síntomas gastrointestinales como el vómito, esta probabilidad aumentaba considerablemente en casi cuatro veces para IAM.

Los factores de riesgo más frecuentemente observados en la población de pacientes infartados fueron los 5 factores de riesgo clásicos: Edad (Hombres $\geq 45$ años; Mujeres $\geq 55$ años), HTA, dislipidemia, sedentarismo y tabaquismo; los cuales han sido descritos desde los años 1980 por varios estudios, recientemente por James L Meisel ${ }^{[6]}$

Tabla 3. Factores asociados a la presencia de Infarto Agudo de Miocardio en los pacientes que consultaron al servicio de urgencias del Hospital Universitario de Neiva

\begin{tabular}{lcccc}
\hline Yariable & OR & IC & CHI2 & P \\
\hline Irradiación del dolor a brazo izquierdo & 2.8 & $1.2-6.4$ & 5.1 & 0.008 \\
Irradiación del dolor a hombro izquierdo & 3.5 & $1.2-10.6$ & 4.1 & 0.01 \\
Intensidad del dolor de 10/ 10 & 2.6 & $1.2-5.9$ & 4.7 & 0.01 \\
Duración del dolor 1-3 horas & 3.8 & $1.4-10.8$ & 5.8 & 0.005 \\
Vómito & 3.8 & $1.4-9.9$ & 7.0 & 0.002 \\
Edad (H>45 años; $M>55$ años) & 7.7 & $1.7-34.2$ & 8.0 & 0.0006 \\
Tabaquismo & 2.6 & $1.2-5.6$ & 5.7 & 0.005 \\
\hline
\end{tabular}


Incidencia y factores asociados para infarto agudo de miocardio en pacientes con dolor torácico/Leidy Henao P., et al.

Dentro de los factores para enfermedad cardiovascular, los que fueron encontrados como significativos para su asociación con IAM fueron la edad (Hombres $\geq 45$ años; Mujeres $\geq 55$ años) y el tabaquismo. La expectativa de vida a nivel mundial ha aumentado y sigue en crecimiento debido a los cambios revolucionarios en la medicina, el cuidado y el control de las enfermedades. Esto permite que la población envejezca y por lo tanto con ello aumente también la aparición de diversas enfermedades crónicas, como por ejemplo la enfermedad coronaria. Tal como lo plantea RODRIGUEZ, Lourdes y col. ${ }^{[7]}$ en su estudio; en el cual muestra que la mayor incidencia de IAM se presenta en pacientes de género masculino con una relación de 2:1 con respecto al género femenino en pacientes de 70 años o más. Este aumento en la expectativa de vida, acompañada de la occidentalización que se ha venido presentando a nivel mundial permite que los hombres de 45 años o más y mujeres de 55 años o más, eleven el riesgo de cursar con IAM en casi ocho veces más como lo muestra nuestro estudio.

En el estudio estandarizado de casos y controles INTER$\mathrm{HEART}^{[8]}$, que incluyó a población de 52 países en representación de todos los continentes, el cual se realizó con el objetivo de evaluar la asociación entre los factores de riesgo potencialmente modificables e infarto agudo de miocardio (IAM), mostró como resultado que el tabaquismo tiene un OR de 2,87, En este presente estudio se encontró un valor significativo muy similar, ya que el tabaquismo se asoció con un aumento de la probabilidad de IAM en 2,6 veces en comparación con personas no fumadoras.

En el estudio de Mora Guillermo y colaboradores ${ }^{[9]}$ se estudiaron las características clínicas y paraclínicas de los pacientes con dolor de pecho. Se tomaron 398 pacientes de una unidad de dolor torácico encontrando que el 29,4\% tenían diagnóstico de infarto agudo de miocardio, el 29,9\% angina inestable y el reto era de origen no isquémico. El $54,6 \%$ de los pacientes pertenecían al género masculino, la edad media fue de 62,3 años; el dolor de ubicación retro-esternal, precordial, con irradiación a cuello asociado de nauseas, vómito y diaforesis fueron los síntomas que mostraron relación estadística con la presencia de infarto de miocardio. La diabetes mellitus y la dislipidemia mostraron fuerte asociación con un OR: 2,14 p: <0,001 para diabetes y OR: 1,8 p: $<0,001$ para dislipidemia. Nuestro estudio muestra hallazgos generales similares, sin embargo los síntomas y hallazgos clínicos que muestran asociación difieren excepto por el vómito. Los relacionados en nuestra investigación son: la duración de más de 1 hora, la intensidad del dolor de 10/10 y la irradiación a hombro y brazo izquierdo. El tabaquismo como factor de riesgo en el estudio de referencia no muestra asociación, sin embargo al combinarlo con otras entidades de riesgo como hipertensión arterial sistólica, diabetes mellitus y dislipidemia aumenta la probabilidad de síndrome coronario agudo; nuestro estudio difiere de este dato ya que encontramos que la presencia de tabaquismo como antecedente tiene un OR de 2,6 para infarto de miocardio ( $\mathrm{p}: 0,005$ ). Lo anterior destaca la pluralidad de los hallazgos clínicos de los pacientes con síndrome coronario agudo.

Dentro del tratamiento de egreso de los pacientes con IAM en este estudio se encontró que de manera correcta y tal como lo recomiendan las guías clínicas, al 100\% se le prescribió ASA; sin embargo una terapia que se ha observado que mejora la sobrevida de los pacientes como lo son los Betabloqueadores no fue prescrita como tratamiento ambulatorio en el total de la población, aunque estos pacientes representaron un pequeño porcentaje (aproxi- madamente $3 \%$ ). A la mayoría de los pacientes, se les prescribió IECAs y estatinas. Para sorpresa de los investigadores, se encontró que aproximadamente un tercio de los pacientes infartados egresaban sin doble antiagregación (adicionando al ASA el Clopidogrel).

Sprockel John Jaime y colaboradores ${ }^{[10]}$ publicaron un estudio sobre las características clínicas de los pacientes con síndrome coronario agudo en los pacientes de medicina interna del Hospital San José entre 1 de Enero del 2009 hasta 31 de Diciembre del 2010. De un total de 133 pacientes, e 63\% eran hombres y la media de edad fue de 64,8 años, hallazgos similares a los de nuestra investigación. El infarto agudo de miocardio con elevación del ST fue del $45,9 \%$ y sin elevación del ST fue del 39\%, la angina inestable fue de $15,1 \%$. Nuestra investigación difiere de esta relación debido a que los pacientes con elevación del ST fueron los de menor proporción, esta puede ser explicado porque en el estudio de referencia se toman solo los pacientes con síndrome coronario confirmado y el de nosotros toda la población con dolor torácico. En el tratamiento el $93 \%$ de los pacientes recibieron betabloqueadores, el $88 \%$ inhibidores de la enzima convertidora de angiotensina y el $97 \%$ antiagregantes plaquetario, estos datos son muy parecidos a los de nuestra investigación. La mortalidad fue del 8,7\% dado similar al de nuestro. Se trombolizo el $82 \%$ de los pacientes de ventana; nuestro estudio no midió este parámetro. E1 tratamiento instaurado a nuestros pacientes se encuentra en los parámetros nacionales.

\section{Limitaciones}

El reducido tamaño de la muestra hace parte de las limitaciones que pudieran no permitir de manera certera aplicar estos resultados a otras poblaciones.

\section{Conclusiones}

La presentación del IAM es más frecuente en la población del género masculino observándose una relación de 2:1 frente al género femenino, siendo en ambos grupos más común la presentación en pacientes añosos, de la zona urbana.

La mayoría de los pacientes con IAM presentan un cuadro clínico típico compatible con las características descritas en la literatura mundial para síndrome coronario agudo. Características clínicas como: irradiación del dolor a brazo y hombro izquierdo, duración del dolor de 1- 3 horas, intensidad máxima del dolor en la escala subjetiva del mismo y la asociación con síntomas como el vómito son estadísticamente significativas en su asociación con IAM en los pacientes que consultan por dolor torácico y/o equivalente anginoso. Factores como la edad $\geq 45$ años en hombres y $\geq 55$ años en mujeres y el tabaquismo aumentaban el riesgo de IAM en 7,7 y 2,6 veces respectivamente.

\section{Referencias}

1. Vidar R, Mathisen M. Prevalence and prognosis of nonspecific chest pain among patients hospitalized for suspected acute coronary syndrome a systematic literature search. BMC Medicine 2012;(10):1-8. 
R.F.S Revista Facultad de Salud

Julio - Diciembre de 2014;6(2): 32-38
Incidencia y factores asociados para infarto agudo de miocardio en pacientes con dolor torácico / Leidy Henao P., et al.
2. Berwanger $O$, Polanczyk $C A$, Rosito $G$. Chest pain observation units for patients with symptoms suggestive of acute cardiac ischaemia. The Cochrane Library 2009, Issue 1 .

3. Dimitrios AS, Dimitrios N. Tziakas GC. Chest Pain in Patients with Arterial Hypertension, Angiographically Normal Coronary Arteries and Stiff Aorta: The Aortic Pain Syndrome. Hellenic Journal Cardiology 2013; 54: 25-31.

4. Bassand JP, Stefan A. Guía de práctica clínica de la ESC para el manejo del síndrome coronario agudo en pacientes sin elevacion persistente del segmento ST. Revista Española de Cardiología. 2012;65(2):173.el-e55.

5. Swap CJ, Nagurney JT. Value and Limitations of Chest Pain History in the Evaluation of Patients With Suspected Acute Coronary Syndromes. The Journal of the American Medical Association: JAMA. 2005;294:2623-2629.

6. Meise, JL. Diagnostic approach to chest pain in adults. En: Visión morfológica. Uptodate. Enero 18, 2011.
7. Rodríguez L, Herrera GV Algunos factores de riesgo que favorecen la aparición del Infarto agudo de miocardio. Revista Cubana Medicina General Integral $1998 ; 14(3): 243-249$

8. Yusuf, Salim et al. Effect of potentially modifiable risk factors associated with myocardial infarction in 52 countries (the INTERHEART study): case-control study. Lancet. September 11, 2004; Vol. 364.

9. Mora $G$, Franco R, Fajardo $H$, et al. Características clínicas y electrocardiográficas de los pacientes que ingresan a una unidad de dolor torácico en el contexto de la nueva definición de infarto agudo de miocardio. Revista Colombiana de Cardiología. 2005; 1 1:333-343.

10. Sprockel JJ, Diaztagle JJ, et al. Descripción Clínica y Tratamiento de los pacientes con síndrome coronario agudo. Acta Médica Colombiana, 2014;39(2):124-130. 